\title{
High-Energy Continuum of Magnetic Excitations in the Two-Dimensional Quantum Antiferromagnet $\mathrm{Sr}_{2} \mathrm{CuO}_{2} \mathrm{Cl}_{2}$
}

\author{
K. W. Plumb, ${ }^{1}$ A. T. Savici, ${ }^{2}$ G. E. Granroth, ${ }^{2,3}$ F.C. Chou, ${ }^{4}$ and Young-June Kim ${ }^{1}$ \\ ${ }^{1}$ Department of Physics, University of Toronto, Toronto, Ontario, Canada M5S $1 A 7$ \\ ${ }^{2}$ Neutron Data Analysis and Visualization Division, \\ Oak Ridge National Laboratory, Oak Ridge, Tennessee 37831, USA \\ ${ }^{3}$ Quantum Condensed Matter Division, Oak Ridge National Laboratory, Oak Ridge, Tennessee 37831, USA \\ ${ }^{4}$ Center for Condensed Matter Sciences, National Taiwan University, Taipei, 10617 Taiwan
}

(Dated: October 12, 2018)

\begin{abstract}
We have measured the magnetic excitation spectrum of the model square-lattice spin- $1 / 2$ antiferromagnet $\mathrm{Sr}_{2} \mathrm{CuO}_{2} \mathrm{Cl}_{2}$ over a broad range of energy and momentum using high-resolution inelastic neutron scattering (INS). The magnon dispersion along the zone boundary was accurately measured to be a $43 \mathrm{meV}$ between $(1 / 2,0)$ and $(3 / 4,1 / 4)$ indicating the importance of coupling beyond nearest neighbors in the spin Hamiltonian. We observe a strong momentum dependent damping of the zone-boundary magnons at $(1 / 2,0)$ revealing a high energy continuum of magnetic excitations. A direct comparison between our INS measurements and resonant inelastic X-ray scattering (RIXS) measurements shows that the RIXS spectrum contains significant contributions from higher energy excitations not previously considered. Our observations demonstrate that this high-energy continuum of magnetic fluctuations is a ubiquitous feature of insulating monolayer cuprates, apparent in both inelastic neutron and light scattering measurements.

PACS numbers: 78.70.Nx,75.30.Ds
\end{abstract}

In the continued search for new states of matter, characterized by unconventional collective modes, model systems play an essential role advancing quantitative understanding of novel theoretical ideas and state of the art experimental techniques. The value of a well-established model system is that it allows researchers to focus on solving, rather than defining, the problem. The spin- $1 / 2$ Heisenberg model on a square lattice is one of the most extensively studied systems of quantum magnetism. Interest in this model has been largely stimulated by its relevance to the parent compounds of the high temperature cuprate superconductors 1 , $\mathrm{La}_{2} \mathrm{CuO}_{4}$ is perhaps the best known example in this regard 2. While the longwavelength spin excitations in $\mathrm{La}_{2} \mathrm{CuO}_{4}$ are correctly described by a renormalized spin wave theory within the $2 \mathrm{D}$ Heisenberg model, experiments probing the high energy spin fluctuations have shown that the nearest-neighbor Heisenberg model cannot capture details of the spin excitation spectrum at short wavelengths. For example, the broad asymmetrical line-shape of the two-magnon Raman scattering cannot be explained by calculations based on spin-wave theory 3 , 4. More recently, inelastic neutron scattering (INS) measurements conducted by Headings et al. reveal a high energy momentum dependent continuum of magnetic excitations in $\mathrm{La}_{2} \mathrm{CuO}_{4}$ [5].

In this letter, we report high resolution INS measurements of the magnetic excitation spectrum of another well-known 2D model system, the insulating cuprate $\mathrm{Sr}_{2} \mathrm{CuO}_{2} \mathrm{Cl}_{2}$ [6] 6 . We observe a strong momentum dependent damping of the zone boundary magnons indicating the presence of a high-energy continuum of magnetic excitations in $\mathrm{Sr}_{2} \mathrm{CuO}_{2} \mathrm{Cl}_{2}$. Furthermore, we directly compare the INS spectra with previously reported res- onant inelastic X-ray scattering (RIXS) results [9]. Importantly, the RIXS and INS measurements are in agreement over a large portion of the Brillouin zone except in the neighborhood of $(1 / 2,0)$ where RIXS overestimates the zone boundary energy by $\sim 25 \mathrm{meV}$. This deviation occurs in precisely the region of phase-space where the continuum scattering carries significant spectral weight. The strong coupling of the RIXS cross-section to the momentum dependent continuum, in conjunction with the broad energy resolution of RIXS, accounts for discrepancies between the zone-boundary magnon dispersion measured by RIXS and INS. Thus, the high-energy momentum dependent continuum of spin fluctuations previously observed in $\mathrm{La}_{2} \mathrm{CuO}_{4}$ [5] appears to be a general feature of the magnetic excitation spectrum in the parent cuprates, evident in both inelastic neutron and light scattering measurements.

For our measurements, large single crystals of $\mathrm{Sr}_{2} \mathrm{CuO}_{2} \mathrm{Cl}_{2}$ were grown from flux following identical synthesis procedures to previous reports [10. $\mathrm{Sr}_{2} \mathrm{CuO}_{2} \mathrm{Cl}_{2}$ has a tetragonal unit cell, space group $\mathrm{I} 4 / \mathrm{mmm}$, down to at least $5 \mathrm{~K}$ with lattice parameters, a $=\mathrm{b}=3.96 \AA$ and $\mathrm{c}=15.53 \AA$ and we index momentum transfer $\mathbf{Q}=h \mathbf{a}^{\star}+k \mathbf{b}^{\star}+l \mathbf{c}^{\star}$ using the reciprocal lattice of the structural unit cell. Measurements were performed on an array of seven co-aligned single crystals with a total mass of $17 \mathrm{~g}$, and a mosaic spread of $2^{\circ}$. The sample was aligned with the $\mathbf{c}^{\star}$-axis along the incident neutron wavevector, $k_{i}$ for the duration of the measurement.

INS measurements were performed on the fine resolution Fermi chopper spectrometer SEQUOIA, at the Spallation Neutron Source (SNS) at Oak Ridge National Laboratory. Measurements of the zone boundary magnetic 
excitations were carried out in two configurations: with Fermi chopper 1 rotating at a frequency of $480 \mathrm{~Hz}$ phased for an incident energy of $450 \mathrm{meV}$, and with Fermi chopper 1 at $300 \mathrm{~Hz}$ phased for an incident energy of $150 \mathrm{meV}$. A T0 chopper rotating at $120 \mathrm{~Hz}$ was used to eliminate the fast neutron background [11, 12]. INS intensities were put on an absolute scale by normalization with incoherent elastic scattering from the sample.

Figure 1 shows a set of typical constant energy transfer slices in the $(h, k)$ plane of the raw neutron scattering intensity. The INS cross-section is directly proportional to the dynamical structure factor and high intensity regions are produced when the spectrometer resolution volume intersects with the spin excitation dispersion surface. In Fig. 1 cones of spin waves can be seen emerging from the antiferromagnetic zone centers at $(1 / 2,1 / 2)$ which disperse isotropically at low energies. As the zone-boundary energy is approached around $280 \mathrm{meV}$ [Fig. 1 (e)], the high intensity regions become localized near the $(1 / 2,0)$ positions in reciprocal space.

More details of the spin excitations can be extracted from constant energy transfer and constant momentum transfer cuts through the dispersion surface. Representative cuts are presented in figure 2. The spin wave dispersion and momentum dependence of intensity were accurately determined by independently fitting a large number of cuts, spanning the full Brillouin zone, with a model cross-section based on the Hamiltonian in equation (1) convolved with the spectrometer resolution function 13. Figures 3 (a) and (b) show the dispersion and spin wave intensities respectively along high symmetry directions obtained from the cuts.

The data at small momentum transfers, close to the magnetic zone center, and over a large portion of the zone boundary are consistent with results from previous low-energy INS measurements [7] as well as the recent RIXS results. We also observe a large, $43 \mathrm{meV}$, dispersion along the zone-boundary, between $(1 / 2,0)$ and $(3 / 4,1 / 4)$; however, this is significantly smaller than the $70 \mathrm{meV}$ zone boundary dispersion reported by a RIXS study on the identical compound [9], but still much larger than the $22 \mathrm{meV}$ dispersion reported for $\mathrm{La}_{2} \mathrm{CuO}_{4}$ [15.

The observed dispersion can be analyzed following previous work on $\mathrm{La}_{2} \mathrm{CuO}_{4}$ which described the excitation spectrum using classical linear spin wave theory and a Heisenberg Hamiltonian including higher order couplings in the spin operator [5, 15]:

$$
\begin{aligned}
H= & J \sum_{\langle i, j\rangle} \mathbf{S}_{i} \cdot \mathbf{S}_{j}+J^{\prime} \sum_{\left\langle i, i^{\prime}\right\rangle} \mathbf{S}_{i} \cdot \mathbf{S}_{i^{\prime}}+J^{\prime \prime} \sum_{\left\langle i, i^{\prime \prime}\right\rangle} \mathbf{S}_{i} \cdot \mathbf{S}_{i^{\prime \prime}} \\
& +J_{c} \sum_{\langle i, j, k, l\rangle}\left\{\left(\mathbf{S}_{i} \cdot \mathbf{S}_{j}\right)\left(\mathbf{S}_{k} \cdot \mathbf{S}_{l}\right)+\left(\mathbf{S}_{i} \cdot \mathbf{S}_{l}\right)\left(\mathbf{S}_{k} \cdot \mathbf{S}_{j}\right)\right. \\
& \left.-\left(\mathbf{S}_{i} \cdot \mathbf{S}_{k}\right)\left(\mathbf{S}_{j} \cdot \mathbf{S}_{l}\right)\right\}
\end{aligned}
$$

where $J, J^{\prime}$, and $J^{\prime \prime}$ are the first, second and third neighbor exchange, and $J_{c}$ is the ring exchange. Expand-

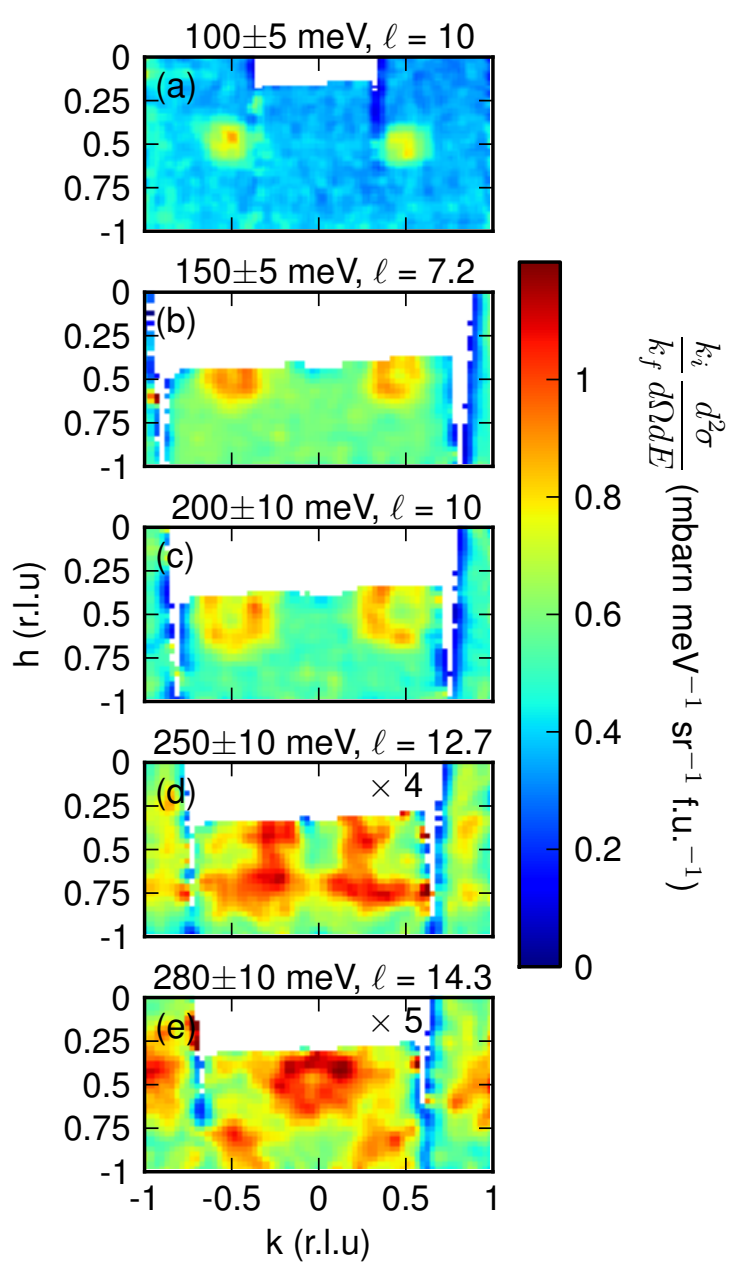

FIG. 1. Representative constant energy transfer slices displaying the raw INS intensity at $\mathrm{T}=5 \mathrm{~K}$. Data in panel (a) was collected with $E_{i}=150 \mathrm{meV}$ and a total proton charge of 47.5 C. Data in panels (b)-(e) was taken with $E_{i}=450 \mathrm{meV}$ and a proton charge of $186 \mathrm{C}$. Data were scaled to absolute units by normalizing with incoherent elastic scattering from the sample.

ing the exchange constants to fourth order in terms of an effective Coulomb repulsion $U$ and nearest neighbor hopping $t$, the exchange constants can be determined in terms of $t$ and $U$ [15, 17. Fitting $t$ and $U$ within linear spin wave theory we obtain $t=0.261(3), U=1.9(5)$ and $J=4 t^{2} / U-24 t^{4} / U^{3}=126(3), J^{\prime}=J^{\prime \prime}=4 t^{4} / U^{3}=2.7(5)$, $J_{c}=80 t^{4} / U^{3}=54(3)$. This fit is shown as a solid black line in Fig. 3 (a). The momentum dependence of the magnon spectral weight also predicted by spin wave theory, including two transverse modes, is compared with the integrated intensity of the one-magnon signal extracted from INS data in Fig. 3 (b). Including an intensity renormalization factor of $Z_{d}=0.48(5)$ [16], there is good agreement with the linear spin wave theory prediction across most of the Brillouin zone except for momentum transfers in near $(1 / 2,0)$ where the measured 


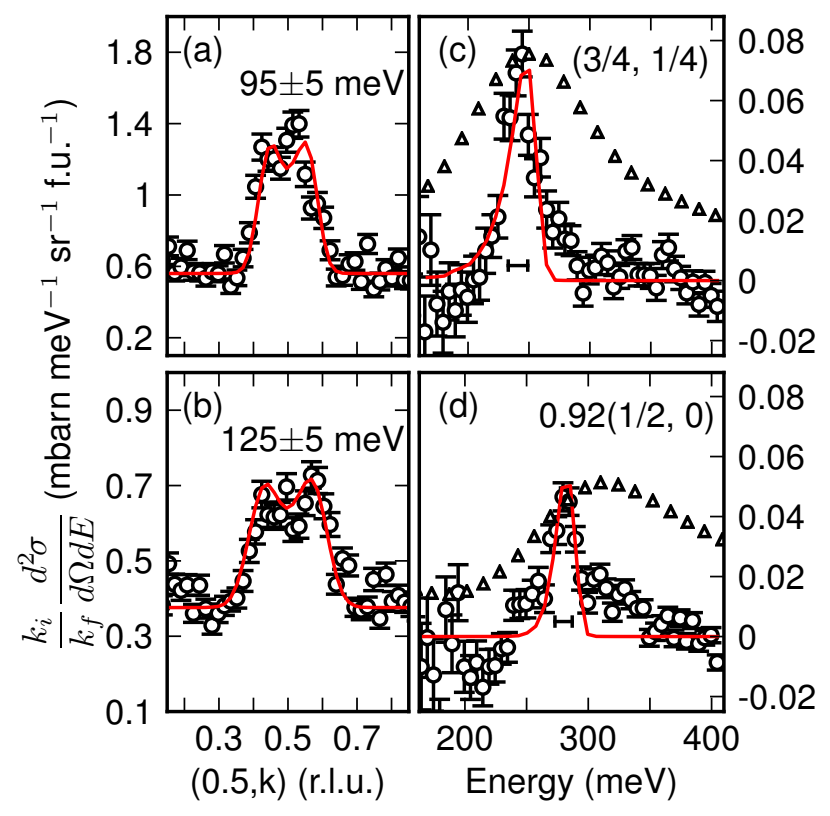

FIG. 2. (a)-(b) Constant energy cuts near the antiferromagnetic zone center for an incident neutron energy of $150 \mathrm{meV}$. (c)-(d) Constant momentum transfer cuts through the zone boundaries integrated over $\pm 0.16 \AA^{-1}$ for an incident neutron energy of $450 \mathrm{meV}$. An energy dependent background, measured near $\mathbf{Q}=(1,0)$ has been subtracted from the constant momentum transfer cuts. Black solid lines show the time-offlight spectrometer resolution FWHM at the zone boundaries. Red lines are fits to the resolution convolved model cross- section, including the anisotropic $\mathrm{Cu}^{2+} d_{x^{2}-y^{2}}$ form-factor [14]. Triangles in (c)-(d) are the corresponding RIXS data from reference 9 .

intensity is strongly suppressed compared with the spin wave theory prediction. The origin of this discrepancy is revealed by the constant momentum transfer cuts which show a high energy tail of scattering forming a momentum dependent continuum around $(1 / 2,0)$. Magnons at $(1 / 2,0)$ couple strongly to these higher energy excitations and are thus heavily damped, reducing the spectral weight contained in the one-magnon peak. The momentum and energy integrated intensity of the inelastic magnetic scattering is $\left\langle M_{\text {inelastic }}^{2}\right\rangle=0.95 \pm 0.30 \mu_{B}^{2}$ corresponding to approximately $65 \%$ of the inelastic spectral weight predicted by renormalized spin-wave theory [16].

An identical suppression of the one-magnon intensity around $(1 / 2,0)$ and momentum dependent continuum scattering has previously been observed in both $\mathrm{La}_{2} \mathrm{CuO}_{4}$ [5] and the square lattice antiferromagnet CFTD [18. The origin of these high energy magnetic excitations is not clear. The broad peak shapes and momentum dependent damping cannot be accounted for by including two-magnon process in the INS crosssection [5, 18. One possible explanation of the high en-

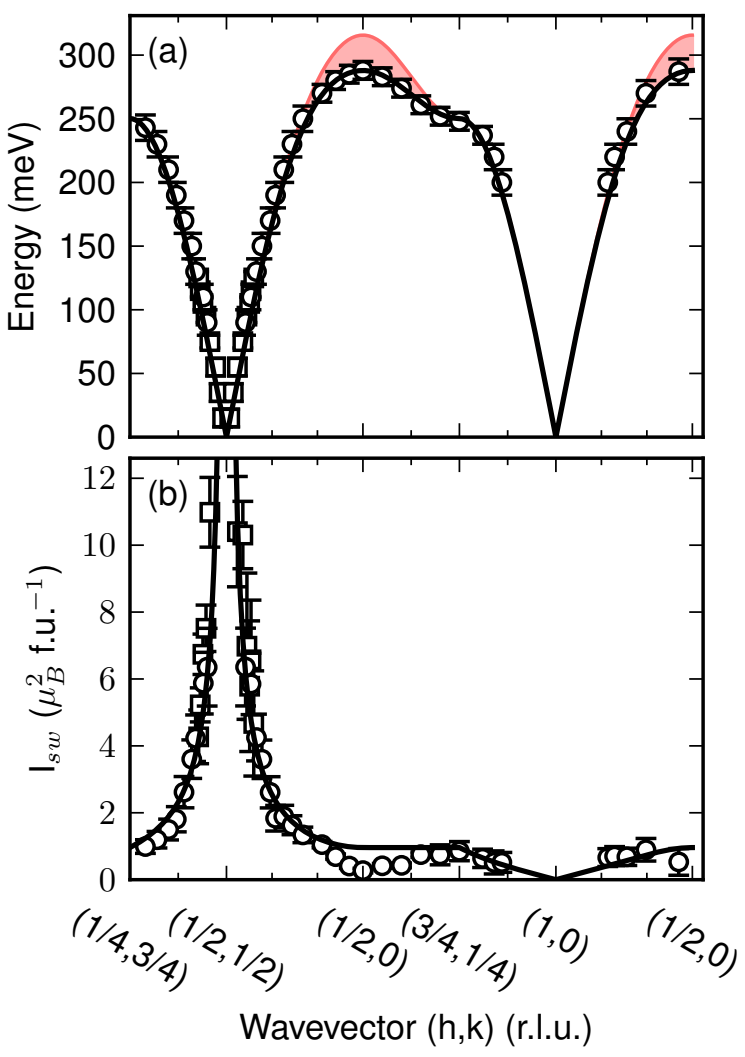

FIG. 3. (a) Magnon dispersion along high symmetry directions measured by INS in $\mathrm{Sr}_{2} \mathrm{CuO}_{2} \mathrm{Cl}_{2}$ at $\mathrm{T}=5 \mathrm{~K}$. Each point was extracted by fitting to individual constant momentum or energy transfer cuts. Square points were obtained from the $E_{i}=150 \mathrm{meV}$ data and the circles from the $E_{i}=450 \mathrm{meV}$ data. The solid black line is the best fit to the spin wave dispersion relation and the red filled area is the difference between the RIXS results of [9] and INS measurement. (b) Wave-vector dependence of the integrated spin wave intensity. The black solid line is the prediction of linear spin wave theory including an intensity renormalization factor $Z_{d}=0.48(5)$ [16].

ergy spectral weight is the existence of spinons with a dwave dispersion, as proposed in some theoretical models of the cuprates [19]. Such high-energy excitations should also be visible in RIXS spectra, which has been shown to couple strongly with the two-and four-spinon continuum present in one-dimensional magnetic systems [20, 21].

RIXS has recently emerged as a powerful probe of collective excitations in quantum condensed matter systems [22]. RIXS has been used to measure the dispersion of charge excitons, orbitons, bimagnons, as well as magnetic excitations in copper oxide based model systems [20, 21, 23, 24. The observation of momentum resolved collective magnetic excitations has generated a great deal of excitement in the research community because RIXS overcomes many challenges of INS. In particular, measurements can be performed on small sam- 
ples, including thin-films [25], and on samples containing highly absorbing elements such as Ir [26]. Unfortunately, coarse energy resolution currently limits the applicability of this technique to only a handful of systems with large magnetic energy scales. RIXS also couples strongly to other types of excitations in addition to magnetic collective modes 22 which, in conjunction with the coarse energy resolution, can severely limit interpretation of experimental data. Since the cross-section of INS is well understood, a detailed comparison of magnetic excitation spectrum measured by RIXS and INS throughout the full Brillouin zone would be of great utility in the development of our understanding of the RIXS cross-section. An earlier comparison for $\mathrm{La}_{2} \mathrm{CuO}_{4}$ found that the magnon spectrum of $\mathrm{La}_{2} \mathrm{CuO}_{4}$ extracted from RIXS agrees with neutron scattering data [15] over most of the Brillouin zone [27, 28]. However, this comparison was limited in momentum space coverage and did not include the zone boundary.

In figure 2 (c) and (d) the corresponding RIXS spectra from reference [9] are superimposed with the INS data. While the position of the RIXS and INS peak intensity is in excellent agreement near $(3 / 4,1 / 4)$, there is a significant $\sim 25 \mathrm{meV}$ discrepancy near $(1 / 2,0)$, [Fig. 2 (d)]. The momentum dependence of this discrepancy is highlighted by the red shaded area in Fig. 3 (a) which shows the difference between the magnon dispersion predicted using parameters extracted from the RIXS data in 9] and the INS result.

In order to extract the spin wave dispersion, RIXS spectra are typically analyzed including contributions from one-and two-magnon scattering. Scattering by the creation of a single magnon is considered to be the dominant process and the energy position of the spectra maximum corresponds closely to the magnon energy [9, 28. Upon comparison with the INS lineshape, it is clear that the simple interpretation of the RIXS spectrum in terms of one-and two-magnon excitations alone is not complete and that there must be a significant higher energy contribution to the RIXS magnetic spectral weight near $(1 / 2,0)$. This was not observed in previous comparisons of RIXS and INS measurements of the magnon dispersion in $\mathrm{La}_{2} \mathrm{CuO}_{4}$ because the RIXS study only extended up to $\mathbf{q} \approx 0.8(1 / 2,0)[28$, just outside the region where we observe the discrepancy between RIXS and INS.

The results presented here show that the momentum dependent continuum scattering at $(1 / 2,0)[5$ is a general feature of the monolayer parent cuprate compounds and that the magnetic excitation spectrum cannot be fully described by a renormalized spin wave theory from an ordered antiferromagnet. This conclusion is further supported by optical measurements where the deficiency of spin wave theory for describing the short wavelength magnetic excitation spectra is particularly apparent. Specifically, spin wave theory fails to explain the asymmetrical line-shape of the two-magnon Raman scattering [3, 4] and high energy spectral weight extending between 400 and $750 \mathrm{meV}$ observed in mid-infrared (midIR) optical absorption measurements 29. MidIR optical absorption spectra of the cuprates show a sharp resonance peak near $400 \mathrm{meV}$ 29] which has been identified as resulting from a bimagnon plus phonon excitation [30; however, the significant spectral weight extending from the $400 \mathrm{meV}$ resonance up to $750 \mathrm{meV}$ cannot be explained in this context. Similarly, through careful analysis of the optical conductivity line-shape in $\mathrm{YBa}_{2} \mathrm{Cu}_{3} \mathrm{O}_{6}$ Grüninger et al. conclude that, while the main resonance peak observed near $400 \mathrm{meV}$ is well described by the bimagnon plus phonon process, the significant spectral weight extending to $750 \mathrm{meV}$ cannot be accounted for within spin wave theory 31. The inclusion of ring exchange and phonon damping terms has also failed to account for the asymmetric Raman scattering line shape [4. A complete description of magnetic interactions in the parent cuprates must account for this high energy, momentum dependent, continuum of magnetic excitations.

In conclusion, we have measured the magnetic excitation spectrum of the model square-lattice spin-1/2 antiferromagnet $\mathrm{Sr}_{2} \mathrm{CuO}_{2} \mathrm{Cl}_{2}$ throughout the full Brillouin zone. Our results show that the high energy momentum dependent continuum of magnetic excitations observed in $\mathrm{La}_{2} \mathrm{CuO}_{4}$ is a general feature of monolayer cuprates. A direct comparison between the magnetic excitation spectra measured by INS and by RIXS reveals significant contributions from higher energy excitations in the RIXS spectra that were not fully considered in the previous analysis. This resulted in an overestimation of the zoneboundary energy at $(1 / 2,0)$. As the energy resolution and theoretical understanding of the RIXS cross-section continues to improve RIXS may become an ideal probe of this high energy momentum dependent continuum of excitations.

We would like to thank John Hill, Mark Dean, and Jeroen van den Brink for thoughtful comments and critical reading of this manuscript. We are also thankful to Michel Gingras for insightful discussions. Work at the University of Toronto was supported by NSERC of Canada. Work at Oak Ridge National Laboratory's Spallation Neutron Source was sponsored by the Scientific User Facilities Division, Office of Basic Energy Sciences, U.S. Department of Energy. K.W. Plumb acknowledges the support of the Ontario Graduate Scholarship.

1] P. A. Lee, N. Nagaosa, and X.-G. Wen, Rev. Mod. Phys. 78, 17 (2006).

[2] M. A. Kastner, R. J. Birgeneau, G. Shirane, and Y. Endoh, Rev. Mod. Phys. 70, 897 (1998).

[3] S. Sugai, M. Sato, T. Kobayashi, J. Akimitsu, T. Ito, H. Takagi, S. Uchida, S. Hosoya, T. Kajitani, and 
T. Fukuda, Phys. Rev. B 42, 1045 (1990).

[4] A. A. Katanin and A. P. Kampf, Phys. Rev. B 67, 100404(R) (2003).

[5] N. S. Headings, S. M. Hayden, R. Coldea, and T. G. Perring, Phys. Rev. Lett. 105, 247001 (2010).

[6] M. Greven, R. J. Birgeneau, Y. Endoh, M. A. Kastner, B. Keimer, M. Matsuda, G. Shirane, and T. R. Thurston, Phys. Rev. Lett. 72, 1096 (1994).

[7] M. Greven, R. J. Birgeneau, Y. Endoh, M. A. Kastner, M. Matsuda, and G. Shirane, Z. Phys. B 96, 465 (1995).

[8] K. R. Thurber, A. W. Hunt, T. Imai, F. C. Chou, and Y. S. Lee, Phys. Rev. Lett. 79, 171 (1997)

[9] M. Guarise, B. Dalla Piazza, M. Moretti Sala, G. Ghiringhelli, L. Braicovich, H. Berger, J. N. Hancock, D. van der Marel, T. Schmitt, V. N. Strocov, L. J. P. Ament, J. van den Brink, P.-H. Lin, P. Xu, H. M. Rønnow, and M. Grioni, Phy. Rev. Lett. 105, 157006 (2010).

[10] L. L. Miller, X. L. Wang, S. X. Wang, C. Stassis, D. C. D. C. Johnston, J. Faber, and C.-K. Loong, Phys. Rev. B 41, 1921 (1990).

[11] G. E. Granroth, D. H. Vandergriff, and S. E. Nagler, Physica. B Condensed Matter 385-86, 1104 (2006).

[12] G. E. Granroth, A. I. Kolesnikov, T. E. Sherline, J. P. Clancy, K. A. Ross, J. P. C. Ruff, B. D. Gaulin, and S. E. Nagler, Journal of Physics: Conference Series 251, 012058 (2010).

[13] T. G. Perring, "TOBYFIT," http://tobyfit.isis.rl. ac.uk (2013).

[14] S. Shamoto, M. Sato, J. M. Tranquada, B. J. Sternlieb, and G. Shirane, Phys. Rev. B 48, 13817 (1993).

[15] R. Coldea, S. M. Hayden, G. Aeppli, T. G. Perring, C. D. Frost, T. E. Mason, S. W. Cheong, and Z. Fisk, Phys. Rev. Lett. 86, 5377 (2001).

[16] J. Lorenzana, G. Seibold, and R. Coldea, Phys. Rev. B 72, 224511 (2005).

[17] J.-Y. P. Delannoy, M. J. P. Gingras, P. C. W. Holdsworth, and A.-M. S. Tremblay, Phys. Rev. B 79, 235130 (2009).

[18] N. B. Christensen, H. M. Rønnow, D. F. McMorrow, A. Harrison, T. G. Perring, M. Enderle, R. Coldea, L. P. Regnault, and G. Aeppli, Proc. Natl. Acad. Sci. 104, 15264 (2007).

[19] C.-M. Ho, V. N. Muthukumar, M. Ogata, and P. W. Anderson, Phys. Rev. Lett. 86, 1626 (2001).
[20] S. Glawion, J. Heidler, M. W. Haverkort, L. C. Duda, T. Schmitt, V. N. Strocov, C. Monney, K. J. Zhou, A. Ruff, M. Sing, and R. Claessen, Phys. Rev. Lett. 107, 107402 (2011)

[21] J. Schlappa, K. Wohfeld, K. Zhou, M. Mourigal, V. N. Haverkort, M. W. amd Strocov, L. Hozoi, C. Monney, S. Nishimoto, S. Singhn, A. Revcolevschi, J. S. Caux, L. Patthey, H. M. Rønnow, J. van den Brink, and T. Schmitt, Nature 485, 82 (2012).

[22] L. J. P. Ament, M. van Veenendaal, T. P. Devereaux, J. P. Hill, and J. van den Brink, Rev. Mod. Phys. 83, 705 (2011)

[23] J. P. Hill, G. Blumberg, Y.-J. Kim, D. S. Ellis, S. Wakimoto, R. J. Birgeneau, S. Komiya, Y. Ando, B. Liang, R. L. Greene, D. Casa, and T. Gog, Phys. Rev. Lett. 100, 097001 (2008)

[24] J. Schlappa, T. Schmitt, F. Vernay, V. N. Strocov, V. Ilakovac, B. Thielemann, H. M. Rønnow, S. Vanishri, A. Piazzalunga, X. Wang, L. Braicovich, G. Ghiringhelli, C. Marin, J. Mesot, B. Delley, and L. Patthey, Phys. Rev. Lett. 103, 047401 (2009)

[25] M. P. M. Dean, R. S. Springell, C. Monney, K. J. Zhou, J. Pereiro, I. Božović, B. Dalla Piazza, H. M. Rønnow, M. E., J. van den Brink, T. Schmitt, and J. P. Hill, Nat. Matter. 11, 850 (2012)

[26] J. Kim, D. Casa, M. H. Upton, T. Gog, Y.-J. Kim, J. F. Mitchell, M. van Veenendaal, M. Daghofer, J. van den Brink, G. Khaliullin, and B. J. Kim, Phys. Rev. Lett. 108, 177003 (2012)

[27] L. Braicovich, L. J. P. Ament, V. Bisogni, F. Forte, C. Aruta, G. Balestrino, N. B. Brookes, G. M. De Luca, P. G. Medaglia, F. Miletto Granozio, M. Radovic, M. Salluzzo, J. van den Brink, and G. Ghiringhelli, Phys. Rev. Lett. 102, 167401 (2009)

[28] L. Braicovich, J. van den Brink, V. Bisogni, M. M. Sala, L. J. P. Ament, N. B. Brookes, G. M. De Luca, M. Salluzzo, T. Schmitt, V. N. Strocov, and G. Ghiringhelli, Phys. Rev. Lett. 104, 077002 (2010)

[29] J. D. Perkins, R. J. Birgeneau, J. M. Graybeal, M. A. Kastner, and D. S. Kleinberg, Phys. Rev. B 58, 9390 (1998).

[30] J. Lorenzana and G. A. Sawatzky, Phys. Rev. B 52, 9576 (1995).

[31] M. Grüninger, D. van der Marel, A. Damascelli, A. Erb, T. Nunner, and T. Kopp, Phys. Rev. B 62, 12422 (2000). 